No. 10(17)

\title{
THE BULLWHIP EFFECT \\ IN THE AR(1) DEMAND STRUCTURE
}

\section{Zbigniew Michna}

\begin{abstract}
In this note we derive the famous formula of F. Chen, Z. Drezner, J.K. Ryan and D. Simchi-Levi [2000a] for the bullwhip effect measure in a simple two-stage supply chain under the assumption that demands constitute autoregressive structure of order 1. Our approach is a little different than in Chen et. al [2000a] and therefore we obtain the formula as an equality unlike Chen et. al [2000a], where they have it as a lower bound. Moreover, we analyze the bullwhip effect measure formula and in some cases we have different conclusions than in Chen et. al [2000a].
\end{abstract}

Keywords: supply chain, bullwhip effect, order-up-to level policy, demand forecasting, AR(1) structure.

JEL Classification: F15, F23, L22.

DOI: $10.15611 / \mathrm{me} .2014 .10 .04$.

\section{Introduction}

The bullwhip effect (the Forrester effect) was recognized by Forrester [1958] in the middle of the twentieth century. The term 'bullwhip effect' was coined by Procter \& Gamble's management. This phenomenon appears in supply chains as the variance amplification in replenishment orders if one moves up in a supply chain. It is considered harmful because of its consequences which are (see e.g. [Buchmeister et al. 2008]): excessive inventory investment, poor customer service level, lost revenue, reduced productivity, more difficult decision-making, sub-optimal transportation, sub-optimal production etc. In the current state of research, typical five main causes of the bullwhip effect are considered (see e.g. [Lee, Padmanabhan, Whang 1997a; 1997b]): demand forecasting, non-zero lead time, supply shortage, order batching and price fluctuation. To decrease the variance amplification in a supply chain (i.e. to reduce the Forrester effect) we need to identify all

\footnotetext{
Zbigniew Michna

Department of Mathematics and Cybernetics, Wrocław University of Economics, Komandorska Street 118/120, 53-345 Wrocław, Poland.

E-mail: zbigniew.michna@ue.wroc.pl
} 
the factors causing the bullwhip effect and to quantify their impact on the effect. In the recent paper of Michna and Nielsen [2013], another cause of bullwhip is indicated, that is lead time forecasting in the case of stochastic lead times. Moreover Michna and Nielsen [2013] claim that demand signal processing and lead time signal processing are equally important causes of the bullwhip effect. Many papers have studied the influence of different methods of demand forecasting on the Forrester effect such as simple moving average, exponential smoothing, and minimum-mean-squared-error forecasts when demands are independent identically distributed or constitute integrated moving-average, autoregressive process or autoregressivemoving average (see: [Graves 1999; Lee, So, Tang 2000; Chen et. al 2000a; $2000 \mathrm{~b}]$ ). Few articles have investigated the impact of stochastic lead times on the bullwhip effect (see [Duc, Luong, Kim 2008; Kim et al. 2006; Do et al. 2014; Michna, Nielsen 2013; Michna, Nielsen, Nielsen 2013; 2014; Nielsen, Michna, Do 2014). Moreover, Michna and Nielsen [2013] is the only paper that considers lead time forecasting, and its impact on bullwhip is expressed quantitatively. In this article we assume that demands constitute autoregressive structure of order 1 and lead time is constant. Additionally, demands are predicted using the simple moving average method. Finally we rediscover the famous formula of F. Chen, Z. Drezner, J.K. Ryan and D. SimchiLevi [2000a] . In our approach we obtain it as an equality, unlike the result of Chen et. al [2000a] where they obtain a lower bound. We discuss this difference and analyze the bullwhip effect measure formula.

\section{Supply chain model with moving average forecasting method}

We will model a supply chain with two stages, that is one retailer and one supplier. We will assume that the retailer observes demands $D_{t}$ (usually $t$ denotes a number of a time period that is a day or a week and $D_{t}$ is a demand during a period of the same length). We will assume that $\left\{D_{t}\right\}_{t=-\infty}^{\infty}$ constitutes a stationary sequence of random variables with $\mathbf{E} D_{\mathrm{t}}=\mu_{D}$ and $\operatorname{Var} D_{t}=\sigma_{D}^{2}$, and a generic random variable for demands will be denoted by $D$. More precisely, demands constitute the first-order autoregressive process

$$
D_{t}=\mu+\rho D_{t-1}+\varepsilon_{t},
$$

where $\mu>0$ and $|\rho|<1$ and $\left\{\varepsilon_{t}\right\}_{t=-\infty}^{\infty}$ is a sequence of independent identically distributed random variables such that $\mathbf{E} \varepsilon_{t}=0$ and $\operatorname{Var} \varepsilon_{t}=\sigma^{2}$. It is easy to notice that 


$$
\mathbf{E} D_{t}=\mu_{D}=\frac{\mu}{1-\rho} \text { and } \operatorname{Var} D_{t}=\sigma_{D}^{2}=\frac{\sigma^{2}}{1-\rho^{2}} .
$$

Moreover we assume that the lead times are deterministic and are equal to $L$. This is rather an unrealistic assumption in many supply chains but it is acceptable in the first approach to the bullwhip problem. Thus the lead time demand at the beginning of period $t$ is defined as follows

$$
D_{t}^{L}=D_{t}+D_{t+1}+\ldots+D_{t+L-1}=\sum_{i=0}^{L-1} D_{t+i} .
$$

The lead time demand is the quantity which is necessary to place an order. Using eq. (1) one can show

$$
D_{t}=\mu_{D}\left(1-\rho^{k}\right)+\rho^{k} D_{t-k}+\sum_{i=0}^{k-1} \rho^{i} \varepsilon_{t-i} .
$$

If $D_{t+i}$ denotes the forecast for a demand during the period $t+i$ at the beginning of period $t$ (that is after $i$ periods), then employing the moving average forecasting method with the prediction horizon $n$ we get

$$
D_{t+i}=\frac{1}{n} \sum_{k=1}^{n} D_{t-k}
$$

where $D_{t-k} k=1,2, \ldots$ are demands which have been observed by the retailer until the beginning of period $t$. Thus it is natural to define the forecast for a lead time demand as follows

$$
D_{t}^{L}=\sum_{i=0}^{L-1} D_{t+i}=L \cdot \frac{1}{n} \sum_{k=1}^{n} D_{t-k} .
$$

Moreover, in our model the retailer applies a base stock policy that is a simple order-up-to-level inventory policy. Let $S_{t}$ be the inventory position at the beginning of period $t$ (later an order is placed). If the order-up-to-level policy is employed then $S_{t}$ is determined in the following way

$$
S_{t}=D_{t}^{L}+z \sigma_{t}
$$

where

$$
\sigma_{t}^{2}=\operatorname{Var}\left(D_{t}^{L}-D_{t}^{L}\right)
$$

is the variance of the forecast error for the lead time demand and $z$ is the normal $z$-score that specifies the probability that demand is fulfilled by the on-hand inventory and it can be found based on a given service level. In 
practice the term $z \sigma_{t}$ has to protect from large deviations of demands about $D_{t}^{L}$. The definition of $\sigma_{t}^{2}$ in this paper differs from the definition in Chen et. al [2000a]. They use more practical and feasible definition of lead time deviation, that is

$$
\sigma_{t}^{2}=C(L, \rho, n) \frac{1}{n} \sum_{k=1}^{n}\left(D_{t-i}-D_{t-i}^{L}\right)^{2}
$$

which is an estimation of (7) and $C(L, \rho, n)$ is a constant depending on $L, \rho$, $n$ (see [Chen et. al 2000a]). In practice we do not avoid the estimation of (7) but for theoretical reasons we use $\sigma_{t}^{2}$ defined in (7) because it simplifies calculations and is equivalent to the assumption $z=0$, that is the safety loading is zero. Therefore we get an equality unlike Chen et. al [2000a] (see Theorem 1 below) where they obtain it as a lower bound (for $z=0$ their bound is tight). This means that the estimation of lead time demand deviation increases the order variance. Thus using the order-up-to-level policy, the order quantity $q_{t}$ placed at the beginning of period $t$ is

$$
q_{t}=S_{t}-S_{t-1}+D_{t-1} \text {. }
$$

Our main purpose is to find $\operatorname{Var} q_{t}$ and then to calculate the following bullwhip effect measure

$$
B M=\frac{\operatorname{Var} q_{t} / \mathbf{E} q_{t}}{\operatorname{Var} D_{t} / \mathbf{E} D_{t}} .
$$

Proposition 1. The variance of the forecast error for the lead time demand $\sigma_{t}^{2}$ defined in (7) does not depend on $t$.

This easily follows from the stationarity of the sequence $\left\{D_{t}^{L}-D_{t}^{L}\right\}_{t=-\infty}^{\infty}$.

Since the variance of the forecast error for the lead time demand is independent of $t$ we have from equations (6) and (9) that

$$
\begin{gathered}
q_{t}=D_{t}^{L}-D_{t-1}^{L}+D_{t-1} \\
=\frac{L}{n} \sum_{k=1}^{n} D_{t-k}-\frac{L}{n} \sum_{k=1}^{n} D_{t-1-k}+D_{t-1}=\left(1+\frac{L}{n}\right) D_{t-1}-\frac{L}{n} D_{t-1-n} \\
=\left(1+\frac{L}{n}\right) \mu_{D}\left(1-\rho^{n}\right)+\left[\frac{L}{n}\left(\rho^{n}-1\right)+\rho^{n}\right] D_{t-1-n}+\left(1+\frac{L}{n}\right) \sum_{k=0}^{n-1} \rho^{k} \varepsilon_{t-1-k},
\end{gathered}
$$

where in the last equality we used formula (3). 
It is easy to notice that $\mathbf{E D}_{t}=\mathbf{E} q_{t}$, thus we can derive the exact form of the bullwhip effect measure.

Theorem 1. The measure of the bullwhip effect has the following form

$$
B M=\frac{\operatorname{Var} q_{t}}{\operatorname{Var} D_{t}}=1+\left(\frac{2 L}{n}+\frac{2 L^{2}}{n^{2}}\right)\left(1-\rho^{n}\right) .
$$

Proof. By formula (10) we get

$$
\begin{gathered}
\operatorname{Var} q_{t}=\left[\frac{L}{n}\left(\rho^{n}-1\right)+\rho^{n}\right]^{2} \operatorname{Var} D_{t-1-n}+\left(1+\frac{L}{n}\right)^{2} \sigma^{2} \sum_{k=0}^{n-1} \rho^{2 k} \\
=\left[\frac{L}{n}\left(\rho^{n}-1\right)+\rho^{n}\right]^{2} \operatorname{Var} D+\left(1+\frac{L}{n}\right)^{2} \sigma^{2} \frac{1-\rho^{2 n}}{1-\rho^{2}} \\
=\left[\left(1+\frac{L}{n}\right) \rho^{n}-\frac{L}{n}\right]^{2} \operatorname{Var} D+\left(1+\frac{L}{n}\right)^{2}\left(1-\rho^{2 n}\right) \operatorname{Var} D \\
=\left[1+\left(\frac{2 L}{n}+\frac{2 L^{2}}{n}\right)\left(1-\rho^{n}\right)\right] \operatorname{Var} D,
\end{gathered}
$$

which gives the thesis of the theorem.

Formula (11) is the well-known result of Chen et. al [2000a]. To our knowledge it is the first result which quantifies the bullwhip effect as the quotient of variances.

\section{Analysis of the bullwhip effect measure and conclusions}

We get the famous result of Chen et al. [2000a] but in their paper the above formula is a lower bound for the bullwhip effect measure. This is caused by

the estimation (8) of $\sigma_{t}$ and confirms that the forecasting (estimation) of any parameter when placing an order is the main cause of the variance amplification. We need to explain that the lack of forecasting, that is without using any method (hit or miss), is also a forecast but the worst one and this should amplify the order variance most of all. Thus we can claim that the estimation of the safety loading is also a cause of the bullwhip effect and then the effect is at least as in (11).

Unlike the analysis in Chen et al. [2000a], we have different conclusions for even and odd $n$ in the formula. More precisely, if $n$ is large then term $\rho^{n}$ does not contribute much in the bullwhip effect measure even for $|\rho|$ close to one. If $n$ is small and $\rho$ is close to negative one it does matter if $n$ is 
odd or even. We can explain this in the following way, that is an even number of demands employed in the prediction to place an order dampens order variability because they are negatively correlated and for small even $n$ they reduce pairwise. Thus we can conclude that for small $n$ and negatively correlated demands, an even number of demands taken in the simple moving average prediction reduces the bullwhip effect, that is the effect has to be smaller than in the case of an odd number of demands taken in the prediction of demand. Although this reduction of bullwhip can cause the increase of the stock level variability which yields a poor customer service level.

It seems that the best circumstances to manage the bullwhip problem is the case when demands are positively correlated and the correlation is close to one $\rho \approx 1$ (but $\rho<1$ ), then the effect will be negligible if we simply take the last demand to predict demands $(n=1)$. Similarly, we can proceed if demands are negatively correlated and the correlation is close to negative one $\rho \approx-1(\rho>-1)$. Then the best choice for the prediction horizon of demands is $n=2$, but in this situation as we noticed before we can observe another insufficiency in supply chains that is the increase of the stock level variability.

Another interesting case we can observe when $n$ is large, which means that the number of past demand observations taken into the simple average method converges to infinity. In this case the effect disappears which means that the bullwhip effect measure goes to one. In the work of Michna and Nielsen [2013], stochastic lead times are considered which are predicted using the simple moving average method with the delay parameter $m$ of prediction. Then large $n$ does not eliminate the effect because lead time forecasting still causes the effect (see Theorem 1 of Michna and Nielsen [2013]). The bullwhip effect is also eliminated if $\mathrm{L}=0$, that is the lead time is zero (see eq. (11)). However this is obvious and intuitive because the orders are realized immediately and demands are fulfilled on-line and no storehouse is necessary. Obviously this is not possible in practice. In the article of Michna and Nielsen (2013), the demands are independent and identically distributed and lead times are also independent and identically distributed. The formulas in Michna and Nielsen [2013] and (11), coincide if the lead time is deterministic (in [Michna, Nielsen 2013] and $\rho=0$ in (11). 


\section{Acknowledgement}

The author wishes to thank the National Centre of Science for its support under the grant 2012/07/B/HS4/00702.

\section{References}

Buchmeister B., Pavlinjek J., Palcic I., Polajnar A. (2008). Bullwhip effect problem in supply chains. Advances in Production Engineering \& Management 3(1). Pp. 45-55.

Chen F., Drezner Z., Ryan J.K., Simchi-Levi D. (2000a). Quantifying the bullwhip effect in a simple supply chain. Management Science 46(3). Pp. 436-443.

Chen F., Drezner Z., Ryan J.K., Simchi-Levi D. (2000b). The impact of exponential smoothing forecasts on the bullwhip effect. Naval Research Logistics 47(4). Pp. 269-286.

Do N.A.D., Nielsen P., Michna Z., Nielsen I.E. (2014). Quantifying the Bullwhip Effect of Multi-echelon System with Stochastic Dependent Lead Time. Advances in Production Management Systems: Innovative and Knowledge-Based Production Management in a Global-Local World. Pp. 419-426.

Duc T.T.H., Luong H.T., Kim Y.D. (2008). A measure of the bullwhip effect in supply chains with stochastic lead time. The International Journal of Advanced Manufacturing Technology 38(11-12). Pp. 1201-1212.

Forrester J.W. (1958). Industrial dynamics - a major break-through for decisionmaking. Harvard Business Review 36(4). Pp. 37-66.

Graves S.C. (1999). A single-item inventory model for a non-stationary demand process. Manufacturing \& Service Operations Management 1(1). Pp. 50-61.

Kim J.G., Chatfield D., Harrison T.P., Hayya J.C. (2006). Quantifying the bullwhip effect in a supply chain with stochastic lead time. European Journal of Operational Research 173(2). Pp. 617-636.

Lee H.L., Padmanabhan V., Whang S. (1997a). Information distortion in a supply chain: the bullwhip effect. Management Science 43(3). Pp. 546-558.

Lee H.L., Padmanabhan V., Whang S. (1997b). The bullwhip effect in supply chains. Sloan Management Review 38(3). Pp. 93-102.

Lee H.L., So K.C., Tang C.S. (2000). The value of information sharing in a twolevel supply chain. Management Science 46(5). Pp. 626-643.

Michna Z., Nielsen P. (2013). The impact of lead time forecasting on the bullwhip effect. arXiv preprint arXiv:1309.7374.

Michna, Z., Nielsen, I.E., Nielsen, P. (2013). The bullwhip effect in supply chains with stochastic lead times. Mathematical Economics 9(16). Pp. 71-88.

Michna Z., Nielsen P., Nielsen I.E. (2014). The impact of lead time forecasting on the bullwhip effect. arXiv preprint arXiv:1411.4289. 
Nielsen P., Michna Z., Do N.A.D. (2014). An Empirical Investigation of Lead Time Distributions. Advances in Production Management Systems: Innovative and Knowledge-Based Production Management in a Global-Local World. Pp. 435442. 